\title{
Power Line Communications Networking Method Based on Hybrid Ant Colony and Genetic Algorithm
}

\author{
Qianghui Xiao, Huan Jin, Xueyi Zhang* \\ Electrical and Information Engineering College, Hunan University of Technology, Zhuzhou, China \\ Email:*774970930@qq.com
}

How to cite this paper: Xiao, Q.H., Jin, H. and Zhang, X.Y. (2020) Power Line Communications Networking Method Based on Hybrid Ant Colony and Genetic Algorithm. Engineering, 12, 581-590.

https://doi.org/10.4236/eng.2020.128040

Received: June 22, 2020

Accepted: August 17, 2020

Published: August 20, 2020

Copyright $\odot 2020$ by author(s) and Scientific Research Publishing Inc. This work is licensed under the Creative Commons Attribution International License (CC BY 4.0).

http://creativecommons.org/licenses/by/4.0/

\begin{abstract}
When solving the routing problem with traditional ant colony algorithm, there is scarce in initialize pheromone and a slow convergence and stagnation for the complex network topology and the time-varying characteristics of channel in power line carrier communication of low voltage distribution grid. The algorithm is easy to fall into premature and local optimization. Proposed an automatic network algorithm based on improved transmission delay and the load factor as the evaluation factors. With the requirements of QoS, a logical topology of power line communication network is established. By the experiment of MATLAB simulation, verify that the improved Dynamic hybrid ant colony genetic algorithm (DH_ACGA) algorithm has improved the communication performance, which solved the QoS routing problems of power communication to some extent.
\end{abstract}

\section{Keywords}

Power Line Carrier, Communication Network, Quality of Service,

Hybrid Ant Colony and Genetic Algorithm

\section{Introduction}

LV-PLC (Low-voltage power line carrier communication) technology has become the key and difficult technology for smart grid development [1]. Owing to the LV-PLC network interference, multipath time-varying channel characteristics, high attenuation, and reliability of the communications is not ideal [2]. The previous studies have mainly made improvements from the physical layer and data link layer, and these methods have proved incapable of solving reliability of low-voltage power line communication network. So, we must adopt a higher 
level of network layer routing protocols [3] [4] [5].

The traditional Ant Colony System (ACS) adopts positive feedback and adaptive mechanism to accelerate the convergence speed of the algorithm, but the algorithm is easy to fall into local optimal [6]. The clustering routing algorithm obtains more shortest paths at the cost of expanding the search scope, which increases the search time of the algorithm and reduces the search efficiency of the optimal path [7]. The flooding algorithm participates in communication by taking all nodes in the network, which will increase the network load and greatly reduce the network communication efficiency [8]. But for low voltage power line carrier communication networks with complex physical topology and strong interference, these algorithms have some limitations in solving the optimal problem of network path. Therefore, this paper proposes a dynamic hybrid ant colony genetic algorithm (DH_ACGA) under QoS routing constraints. The proposed algorithm uses channel delay and node load as the evaluation factors of the algorithm's objective optimization function and genetic algorithm's (GA) individual fitness function. And improve the crossover operator, according to the GA algorithm's global searchability and fast convergence characteristics, improve the network path optimization ability.

Starting from the reality of the power communication network topology, this paper simulates the establishment of a logical communication network. Because the algorithm was affected by the density of nodes in the network, communication efficiency, communication distance and other conditions, and taking into account the number of hops, bandwidth, delay, error rate, packet loss rate, interference and other indicators [9] [10]. The DH_ACGA under multi-objective constraints is simulated and compared with the ACS algorithm in networking performance.

\section{The Logical Topology of Network}

The logic topology of power line communication network was designed based on a tree and star network, as shown in Figure 1 and Figure 2.

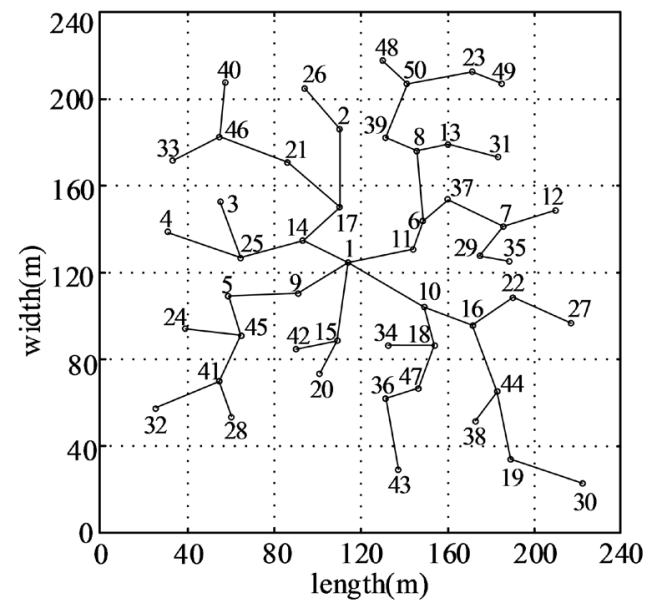

Figure 1. The physical topology of power line communication network. 


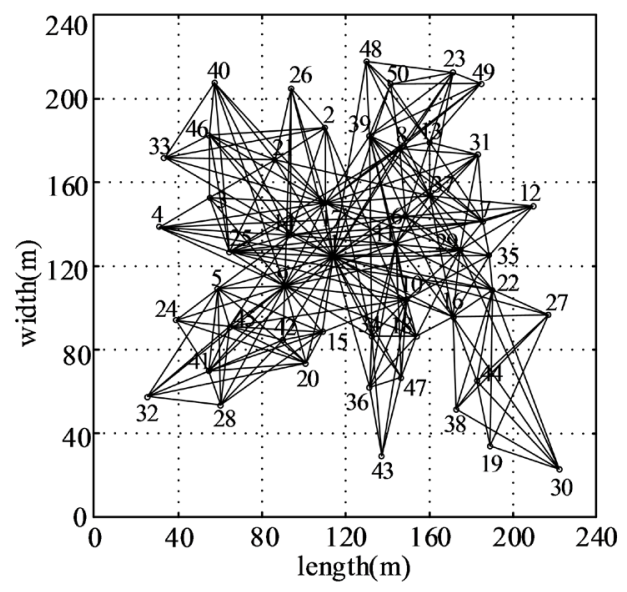

Figure 2. The link logical topology of power line communication network.

Select a power line communication network covering an area of $240 \times 240 \mathrm{~m}^{2}$ in a certain smart grid, and randomly arrange 50 nodes on its secondary side, as shown in Figure 1. Setting node number 1 is the central node, effective communication distance between nodes is $30 \mathrm{~m}$, and 50 nodes available for point-to-point communication, generate a logical topology of Figure 2 as shown. Low power line communication network is a mixed topology, unknown nature of the entire network, and degeneration characteristics of topology. As can be seen from the Figure 2, center node faces maximum number of information transmission, that is, the degree of the node is relatively large [11] [12] [13].

In the real test case, the link connectivity of power line communication network data is very limited in scope. It is not only related to the equipment used in network communication, network load, channel status and other factors, but also related to the degree of the node and the effective communication distance. And when the nodes change, the effects on the logical topology of the entire network is also great.

\section{Hybrid Ant Colony Genetic Algorithm}

Set $\mathrm{m}$ for the numbers of ant, and $\mathrm{n}$ for the number of network communication. The distance between node $i$ and $j$ is $d_{i j}(i, j=1,2, \cdots, n) . \tau_{i j}(t)$ is the pheromone concentrations on the connection path between node $i$ and $j$ at the time of $t$. Initially, the pheromone concentrates on connectivity path is same, so set the $\tau_{i j}(0)=\tau 0$.

\subsection{The Design of Fitness Function}

In the genetic algorithm, the design of fitness function is usually a combination of constraints and the cost function based on the need to meet to construct. But for multi-constrained Quality of Service (QoS) routing, its fitness function can generally be described as $[14]$,

$$
F(z)=\varphi_{1} \cdot g_{\text {delay }}(z)+\varphi_{2} \cdot h_{\text {load }}(z) .
$$


where, $\varphi_{1}, \varphi_{2}$ is the weight of the function, and $\varphi_{1}+\varphi_{2}=1$. It can be adjusted by the values of these two parameters to vary the proportion of the delay and the load node. $g_{\text {delay }}(z)$ is delay function for the network, and its expression is as follows,

$$
g_{\text {delay }}(z)=\frac{\text { delay }_{\text {max }}-\operatorname{delay}(Z(s, d))}{\text { delay }_{\text {max }}}
$$

where,

$$
\operatorname{delay}(Z(s, d))=\sum_{e \in Z(s, d)} \operatorname{delaey}(e) .
$$

In Equations (9) and (10), delay $y_{\max }$ is the maximum network delay, $Z(s, d)$ is the network path to the source node s to the destination node $\mathrm{d}$, delay $(e)$ is the delay of one link e from the source node $s$ to the destination node $d$, and $h_{\text {load }}(z)$ is the load factor of the nodes in the system.

$$
h_{\text {load }}(i)=\frac{1}{\lambda_{i}} .
$$

where, $\lambda_{i}$ is the number of nodes in the network during the transmission of information to be forwarded.

\subsection{The Design of Objective Optimization Function}

Most existing algorithms based on the number of paths to optimize the factors used to evaluate the objective function. But this complex network of distribution network is needed to consider the transmission delay, load balancing, QoS routing algorithm running time, and other factors [15]. To make the system information transfer delay and path load minimum, according to the fitness function, the objective function is expressed as,

$$
\left\{\begin{array}{l}
\min \left(\varphi_{1} \cdot g_{\text {delay }}(z)+\varphi_{2} \cdot h_{\text {load }}(z)\right) \\
\text { s.t } \quad\left\{\begin{array}{l}
|Z(i)-Z(j)| \leq 2 \\
\text { delay }(Z(s, d)) \leq \text { delay }_{\text {max }} \\
h_{\text {load }}(z) \leq h_{\text {max }}
\end{array}\right. \\
Z=\left\{z_{1}, z_{2}, \cdots, z_{N}\right\}
\end{array}\right.
$$

where, $i$ and $j$ represent two adjacent nodes. $Z$ represents the set of carrier nodes of the entire network. $N$ is the number of nodes. $|Z(i)-Z(j)|$ is the distance between two adjacent nodes. The minimum communication distance in the network must be not less than two to ensure communication reliability.

In the case of range of low voltage power line communications network nodes and the complex topology of network, it will undoubtedly lead to information transfer delay, resulting in reduced efficiency of the algorithm processing. The genetic operators are joined in the late of ant colony algorithm, using an improved crossover operator and the mutation operator. after each of iteration, the paths are crossed and mutated, using individual fitness function to select [16]. 
In order to improve the operating efficiency of the algorithm, proposing the fusion conditions of ant colony algorithm and genetic algorithm, and control the switching of iterations and the end of the algorithm through the transmission delay and algorithms. The number of iterations of ant colony algorithm is $N_{a}$. Ant colony algorithm will switch to genetic algorithm at the time of $N a \geq N a_{\max }$, $N a_{\max }$ is maximum number of iterations. Conditions for the end of the whole algorithm system are as follows,

$$
\left\{\begin{array}{l}
N g>N g_{\max } \\
d_{\text {delay }}(Z(s, d))>\text { delay }_{\text {max }}
\end{array}\right.
$$

where, $N_{g}, N g_{\max }, d_{\text {delay }}(\mathrm{Z}(s, d))$, and delay $\max _{\max }$ is the iterations of genetic algorithm, the maximum number of iterations of genetic algorithm, system information transmission delay, and the maximum propagation delay, respectively. When the above conditions are satisfied, output optimal path and end the algorithm. The flow of DH_ACGA shows in Figure 3.

\section{Algorithm Simulation and Feasibility Analysis}

Combined with the logical topology of power line carrier communication network in low voltage distribution network, consider from a fast algorithm, robustness, convergence angles, DH_ACGA algorithm is simulated by MATLAB programming. Test and compare the DH_ACGA algorithm and the ACS algorithm on a simulation model close to the real environment. According to the results of the simulation results, the feasibility of DH_ACGA algorithm is verified.

\subsection{Simulation Parameter Setting}

Using the logical topology of communication networks in Figure 2, the network will be laid 50 communication nodes. Using the ants to find the optimal path of the destination node from the central node is a simulation process. Issue 10 ants from the central node 1 , node 30 represents the ultimate ant destination node and receives the data. The algorithm does not change the value of each node during each iteration. The whole algorithm simulation parameters are showed in Table 1.

\subsection{The Balance of Network Load}

The balance of network load is an important indicator of the reliability of a network. It can be seen from the simulation results in Figure 4, the balance of network load of ACS is poor, the balance of between nodes is inconsistent. Comparing with the ACS algorithm, the network communication load of the DH_ACGA algorithm is significantly reduced. Because the load of nodes in the original ACS algorithm network is not reflected, the difference of the load of network node is relatively large. Some nodes showing high load, overload is likely to cause failure of node communication. The individual fitness value of DH_ACGA algorithm has a great advantage to select the optimum path to balance the load of nodes, so that load balancing is better. 


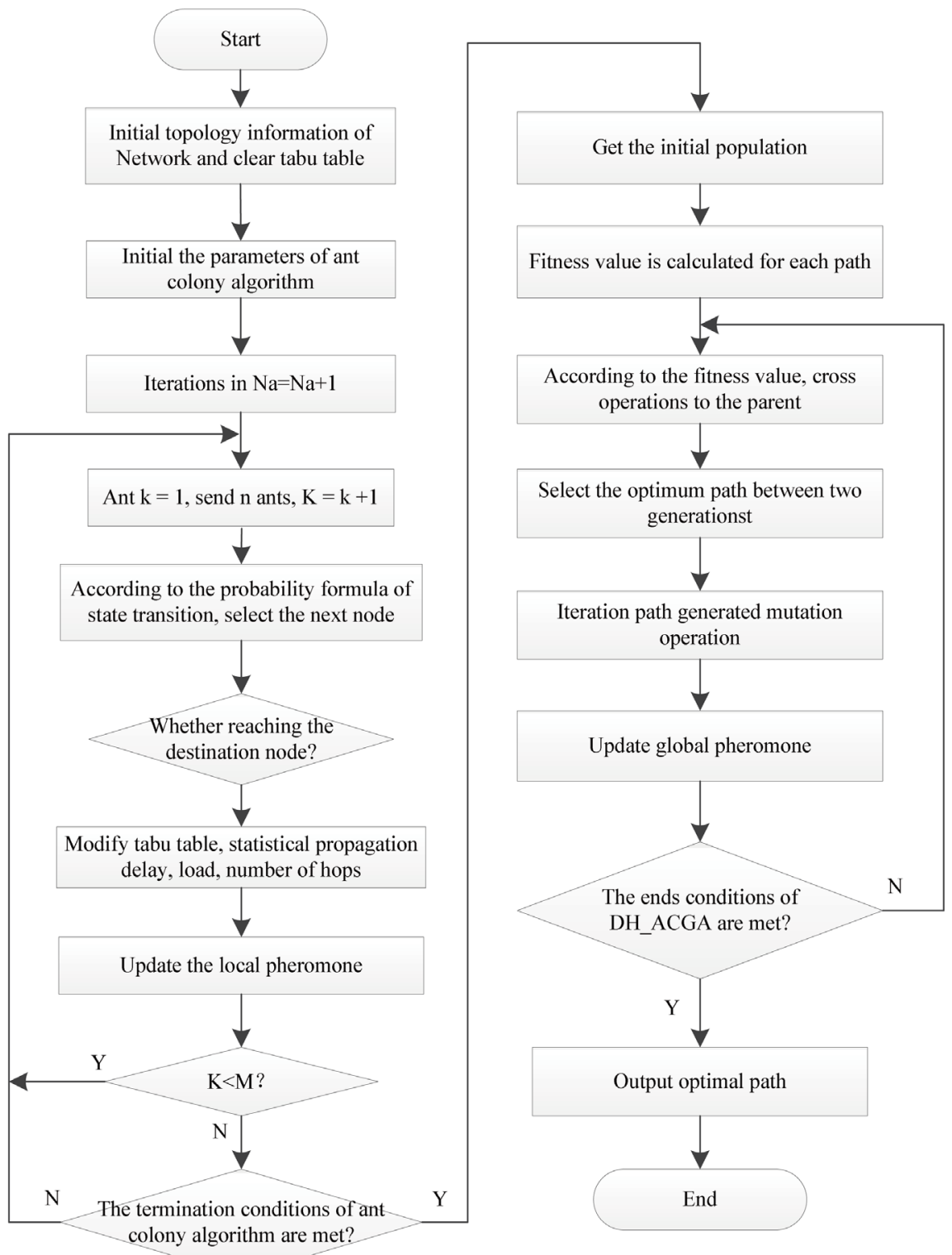

Figure 3. The flow diagram of DH_ACGA.

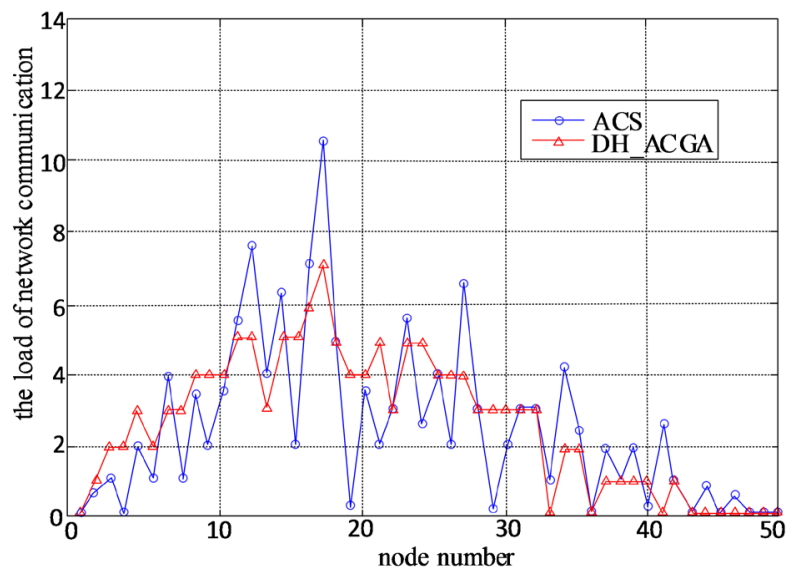

Figure 4. A case of load balancing in PLC network. 
Table 1. The main simulation parameters of experiment.

\begin{tabular}{ccc}
\hline Parameter names & Parameter setting & Parameter meanings \\
\hline$\alpha$ & 50 & The number of nodes in net \\
$\beta$ & 1 & Weighting factor of pheromone \\
$q_{0}$ & 1 & Inspired factor of pheromone \\
$\rho$ & 0.5 & The probability threshold of path searching \\
$\tau_{0}$ & 0.02 & The global pheromone volatilization coefficient \\
$\xi$ & 10 & Initial value of pheromone \\
$\mathrm{Q}$ & 0.04 & The local pheromone volatilization coefficient \\
$\varphi_{1}$ & 100 & The delay weight factor of objective function \\
$\varphi_{2}$ & 0.6 & The load weight factor of objective function \\
$N a_{\max }$ & 0.4 & The maximum number of iterations in ACS \\
\hline
\end{tabular}

\subsection{Hops}

Figure 5 shows the nodes' hops in process of iterative optimization, where, the horizontal axis is the number of iterations of the algorithm, and the vertical axis is each iterative optimization process hops. As can be seen from Figure 5, the DH_ACGA algorithm can converge to the current optimal path (1-16-44-30) after about 16 iterations, while the ACS algorithm demand 20 iterations (1-10-16-38-30). According to the degree of nodes, it can be seen that there are several nodes with relatively high degrees in the optimal path generated by the ACS algorithm.

\subsection{Robustness Analysis of Algorithm}

According to the time complexity and space complexity of the algorithm performance evaluation, the experiment was repeated 50 times respectively. And the statistics of the average convergence value, average convergence iteration number, convergence value, average success rate and calculation time of each algorithm are calculated respectively. The experimental results shown in Table 2 are obtained.

As can be seen from Table 2, ACS algorithm after 19 iterations converges to the optimal path, and DH_ACGA algorithm only need 14 iterations. After several experiments, the average convergence value of the mixture obtained increases, reaching the optimal convergence value indicators required by the system. From the view of the average running time of algorithm, DH_ACGA algorithm compared to the ACS algorithm significantly shortened. It is mainly due to the interaction of crossover and mutation operator, so in the algorithm optimization process, some redundant nodes are avoided, the path optimization time is shorted, and the convergence speed is accelerated. 


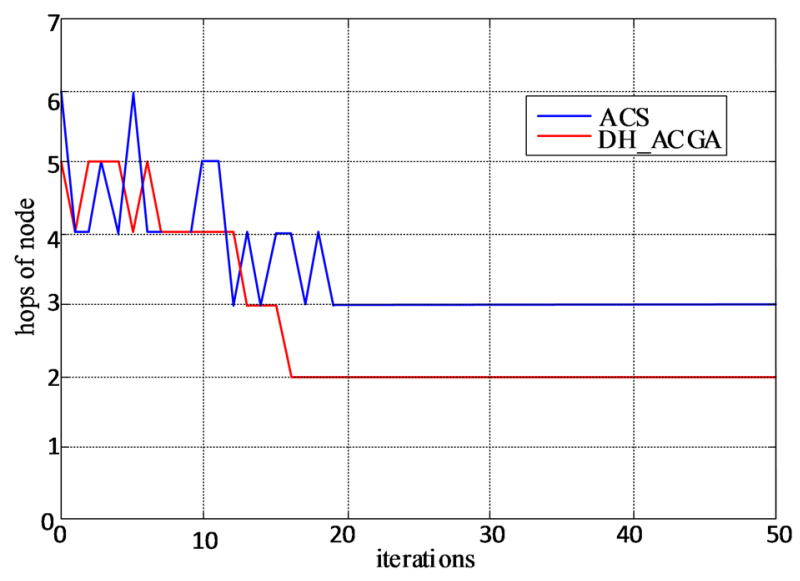

Figure 5. The nodes' hops in process of iterative optimization.

Table 2. Robustness of the algorithm simulation results.

\begin{tabular}{cccccc}
\hline Algorithm & $\begin{array}{c}\text { The number } \\
\text { of nodes in } \\
\text { the optimal } \\
\text { solution }\end{array}$ & $\begin{array}{c}\text { The average } \\
\text { convergence } \\
\text { value }\end{array}$ & $\begin{array}{c}\text { The average } \\
\text { convergence } \\
\text { iterations }\end{array}$ & $\begin{array}{c}\text { Optimal } \\
\text { convergence } \\
\text { value (Optimal } \\
\text { value: } 0.6055)\end{array}$ & $\begin{array}{c}\text { The average } \\
\text { running } \\
\text { time of the } \\
\text { algorithm (s) }\end{array}$ \\
\hline ACS & 8 & 0.6702 & 9 & 0.6811 & 8.35 \\
DH_ACGA & 5 & 0.6010 & 14 & 0.6055 & 5.26 \\
\hline
\end{tabular}

\section{Conclusions}

In this paper, a DH_ACGA algorithm based on delay and load as evaluation factors is proposed on the basis of considering factors such as delay, objective function value, node load, running time, hop count, etc. And compared with the ACS algorithm for simulation and analysis, the following conclusions can be drawn:

1) In the same topology of communications network, analyzing the load balancing of two algorithms, DH_ACGA algorithm can well balance the load of nodes.

2) According to the logical topology of communication nodes in network, sending several "Ant" and setting a target node, DH_ACGA algorithm with a small number of iterations to find the destination node. In the situation of network node failure, information can still reach the target node and has a good self-healing capability of network.

From the simulation analysis of the experiment can prove DH_ACGA algorithm is applicable to LV-PLC network, and be able to provide satisfactory QoS routing services. However, the algorithm also has shortcomings, there is a high complexity degree of time and space. Meanwhile, LV-PLC technology is also faced with the harsh environment, network complexity, noise pollution, electromagnetic interference, and other issues. These issues may lead to lower communication quality and reliability, thus posing a huge challenge to LV-PLC technology. 


\section{Conflicts of Interest}

The authors declare no conflicts of interest regarding the publication of this paper.

\section{References}

[1] Yan, Y.Z. (2018) Analysis of Characteristics of Low Voltage Power Line Carrier Communication Channel. Telecom World, 8, 160-161.

[2] He, X., He, H., Zhou, D., Duan, S.J., Xu, Z.Y. and Ye, X.Q. (2018) Application of Low Voltage Power Carrier Communication Technology in Power Information Acquisition System. Automation and Instrumentation, 10, 190-191+195.

[3] Wang, F., Zhang, S.B. and Wang, Z.C. (2010) Research on Modulation Technology of Low Voltage Power Line Carrier Communication. Video Engineering, 34, 69-72.

[4] Li, Y., Zhang, M. and Zhu, W. (2020) Performance Evaluation for Medium Voltage MIMO-OFDM Power Line Communication System. China Communications, 17, 151-162. https://doi.org/10.23919/JCC.2020.01.012

[5] Tao, L. (2017) Channel Estimation for Power Line Communication. IEEE 17 th International Conference on Communication Technology, Chengdu, 27-30 October 2017, 272-276.

[6] He, F.M., Xu, Y.N., Wang, X.R., Xiong, M.B. and Xiong, Z.H. (2019) An Improved Ant Colony Algorithm for Solving Time-Dependent Road Network Path Planning Problem. 2019 6th International Conference on Information Science and Control Engineering (ICISCE), Shanghai, 20-22 December 2020, 126-130.

[7] Zheng, G., Liu, S. and Qi, X. (2019) Clustering Routing Algorithm and Simulation of Internet of Things Perception Layer Based on Energy Balance. IEEE Access, 7, 145667-145676. https://doi.org/10.1109/ACCESS.2019.2944669

[8] Zhao, M., Ling, Q. and Li, F. (2018) An Iterative Feedback-Based Change Detection Algorithm for Flood Mapping in SAR Images. IEEE Geoscience and Remote Sensing Letters, 16, 231-235. https://doi.org/10.1109/LGRS.2018.2871849

[9] Bumiller, G. and GmbH, I. (2010) Power Line Communication Networks for Large-Scale Control and Automation Systems. IEEE Communications Magazine, 48, 106-113. https://doi.org/10.1109/MCOM.2010.5439083

[10] Zhai, M.Y. (2011) Transmission Characteristics of Low Voltage Distribution Networks in China under the Smart Grids Environment. IEEE Transactions on Power Delivery, 26, 173-180. https://doi.org/10.1109/TPWRD.2010.2067228

[11] Dong, Y.B. and Gao, F. (2003) Analysis of Structure of Carrier Communication Network for Low Voltage Power Line. Power System Technology, 27, 58-62.

[12] Shu, Y., Chen, Q.M. and Li, Y.M. (2003) Predictive Schemes for Future Power Line Communication Part Seven Power Line Communication Network Forms and Their Application. Automation of Electric Power Systems, 27, 90-94.

[13] Wu, Z.H. (2014) Analysis of Low Voltage Power Network Narrowband Power Line Communication Channel Characteristics. Power and Energy, 35, 468-471+474.

[14] Jin, J., Hong, Y., Zhao, F.Q. and Yu, D.M. (2010) Convergence Analysis of Multiple Constrained Routing-Based Ant Colony Optimization Algorithm and Its Application. Control Theory \& Applications, 27, 1353-1361.

[15] Degardin, V., Lienard, M. and Degauque, P. (2003) Optimisation of Equalization Algorithm for Power Line Communication Channel. Electronics Letters, 39, 483-485. https://doi.org/10.1049/el:20030286 
[16] He, W., Zhou, K., Zhang, C., Zhang, X. and Deng, Y. (2014) Study of Channel Capacity for Low Power-Line Based on QoS Strategy. Power System Protection and Control, 42, 106-111. 